\title{
Long-term outcome of primary nephrotic syndrome
}

\author{
O KOSKIMIES, J VILSKA, J RAPOLA, AND N HALLMAN \\ Children's Hospital, University of Helsinki, Finland
}

SUMMARY One hundred and fourteen children with primary nephrotic syndrome were followed up prospectively for periods of between 5 and 14 years. Urine samples from 94 of them became proteinfree during the initial 8-week course of prednisone, and the outcome for these children was good: 74 of them have been free of symptoms for at least 3 years, 18 have had relapses during the last 3 years, and only one child still has proteinuria. All these children have normal renal function and blood pressure. One child died accidentally. Twenty children did not respond to the initial prednisone treatment. Thirteen of them had remissions later, of whom 2 have had relapses during the last 3 years. Seven were totally resistant to prednisone 4 of whom died in renal failure, the remaining 3 have persistent proteinuria with normal levels of creatinine; one has high blood pressure too. Remission during the initial treatment indicated a good prognosis, but two-thirds of the initial non-responders also fared well.

Most children with primary nephrotic syndrome (NS) have minimal changes in renal histopathological specimens. In a survey of 521 children with primary NS, $77 \%$ had minimal changes in the renal biopsy. Physical examination and laboratory tests showed that in the early stage of the disease patients with membranoproliferative (mesangiocapillary) glomerulonephritis could clearly be differentiated from patients with minimal change NS or patients with other histological types of NS. ${ }^{1}$

At least $90 \%$ of children with NS and minimal changes in the histology respond to treatment with prednisone, and about one-third of such patients do not relapse at all after primary remission. However, in one recent series about $40 \%$ patients relapsed frequently and many of them developed signs of steroid toxicity; some required treatment with cytotoxic drugs. ${ }^{2}$

We report the long-term outcome for 114 children who were treated and followed up prospectively at this hospital during the period 1967-76, and relate the result of treatment in the initial illness to our eventual success in controlling the disease. This approach is important because nowadays only a few patients undergo renal biopsy, and the physician has to assess the prognosis from the clinical features. Our patients comprised an unselected series of children with NS, which explains why there were so many clinically and histologically less severe cases compared with many other large series.

\section{Patients and methods}

During a 10-year period, 1967-76, 118 children with primary NS were examined and treated at this hospital. Forty-two were girls. Two went into spontaneous remission during the initial investigation and at no time relapsed, one patient moved abroad and was lost to follow-up, and one patient was treated with cyclophosphamide only. After excluding these 4 patients the series comprised 114 children. These $\mathbf{4 0}$ girls and 74 boys were followed up for between 5 and 14 years and, with the exception of 3 , all of them have been seen at least once a year.

If a diagnosis of NS was confirmed, comprehensive investigations, including renal biopsy, were performed in the hospital. Until the end of 1973 every patient underwent a renal biopsy. Since 1974 each patient has been started on treatment with prednisone, and a renal biopsy has been performed only if he did not respond to the prednisone or if he relapsed.*

NS was defined as: proteinuria $=40 \mathrm{mg} / \mathrm{h}$ per $\mathrm{m}^{2}$, and hypoalbuminaemia $<2.5 \mathrm{~g} / 100 \mathrm{ml}$. Infants with the congenital NS of the Finnish type were excluded. Other reasons for exclusion were NS secondary to systemic diseases or other obvious causes, including drugs and poisoning.

The initial prednisone regimen was $60 \mathrm{mg} / \mathrm{m}^{2}$ a

* Some of the patients have been reported to the International Study of Kidney Disease in Children. 
day given in three doses for 4 weeks followed by $40 \mathrm{mg} / \mathrm{m}^{2}$ a day in three doses for 3 consecutive days for 4 weeks. If a child relapsed, prednisone was given daily until the urine had been protein-free for 3 consecutive days, when intermittent treatment was resumed. ${ }^{3}$ Response to treatment was defined as a reduction in the rate of urinary protein excretion to $4 \mathrm{mg} / \mathrm{m}^{2}$ per hour, or Albustix 0 to a trace for 3 consecutive days.

Many patients were given cytotoxic drugs; in some this was because they relapsed, in others it was because they had persistent proteinuria. Cyclophosphamide was given for periods ranging from 42 to 90 days. Until 1973 the size of the dose (between 1 and $5 \mathrm{mg} / \mathrm{kg}$ a day) was such that it caused granulocytopenia (white blood count $<3.0 \times$ $\left.10^{9} / 1\right)$ but since 1974 a lower dose has been used and for a shorter time (between 1 and $2.5 \mathrm{mg} / \mathrm{kg}$ a day). Four patients were given chlorambucil $(2 \mathrm{mg} / \mathrm{kg}$ a day) for 42 days. Cytotoxic drugs were generally combined with the lower dosage of prednisone.

The biopsy specimen was studied under a dissecting microscope and small pieces were cut with a razor blade from samples that contained plenty of glomeruli so that they could be examined under an electron microscope and the amount of immunofluorescence measured. If the amount of cortical tissue was small, the rest of the biopsy was fixed in Dubosq-Brazil solution for 2-4 hours and transferred to $10 \%$ buffered formalin solution for further processing. The methods used for the light and electron microscopical examinations and for the immunofluorescence study were standard ones.
Paraffin sections cut at $3 \mu \mathrm{m}$ were stained with haematoxylin and eosin, Mallory's trichrome method, periodic acid Schiff, and periodic acid silver methanine. Frozen sections for immunofluorescence were incubated with fluorescein isothiocyanate together with anti-human IgG, IgM, $\mathrm{IgA}$, the $\mathrm{C} 3$ fraction of complement, and fibrin. Thin sections for electron microscopical examination were contrasted with the uranyl acetate and lead nitrate.

\section{Results}

All 114 children had prednisone treatment in the initial phase of the manifestation of the NS. Ninetyfour of them became protein-free during the 8-week course of prednisone but 20 did not (Figure).

\section{Patients who responded to prednisone.}

\section{At onset of disease}

Urine samples from 94 ( 66 boys, 28 girls) became protein-free after the initial 8-week course of prednisone. The mean age of children who responded favourably to glucocorticoids was $3 \frac{1}{2}$ (range $0 \cdot 6-14 \cdot 7$ ) years, whereas the mean age of the 19 children who did not go into remission was 5 (range 1.4-14.7) years.

Eighty-two of these 94 children had renal biopsies and 78 of the samples could be classified by light microscopical examination as minimal change NS in accordance with the definitions given in $1970 .^{4}$ The remaining specimens showed clear histopathological changes: 3 had mesangial proliferative

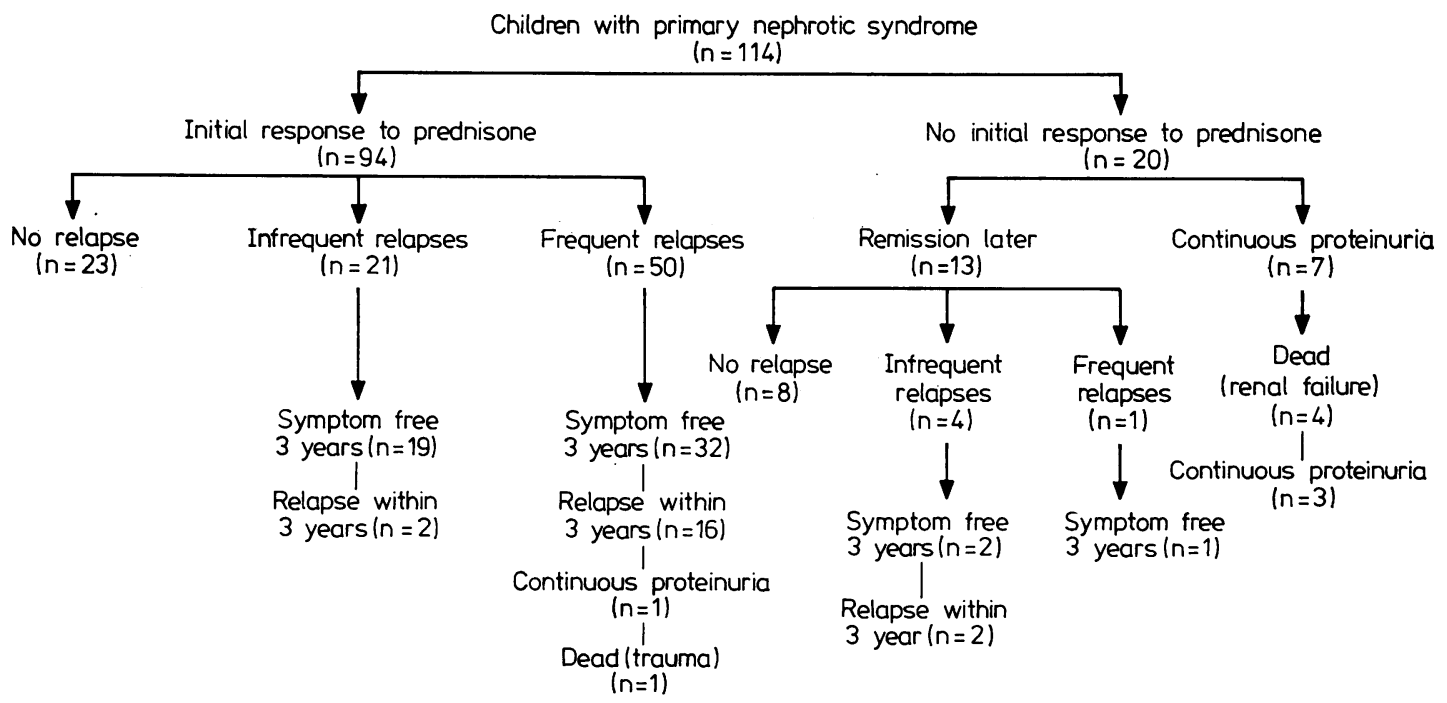

Frequent relapses means at least 2 relapses during any 6-month period

Figure Results of treatment with prednisone in 114 children. 


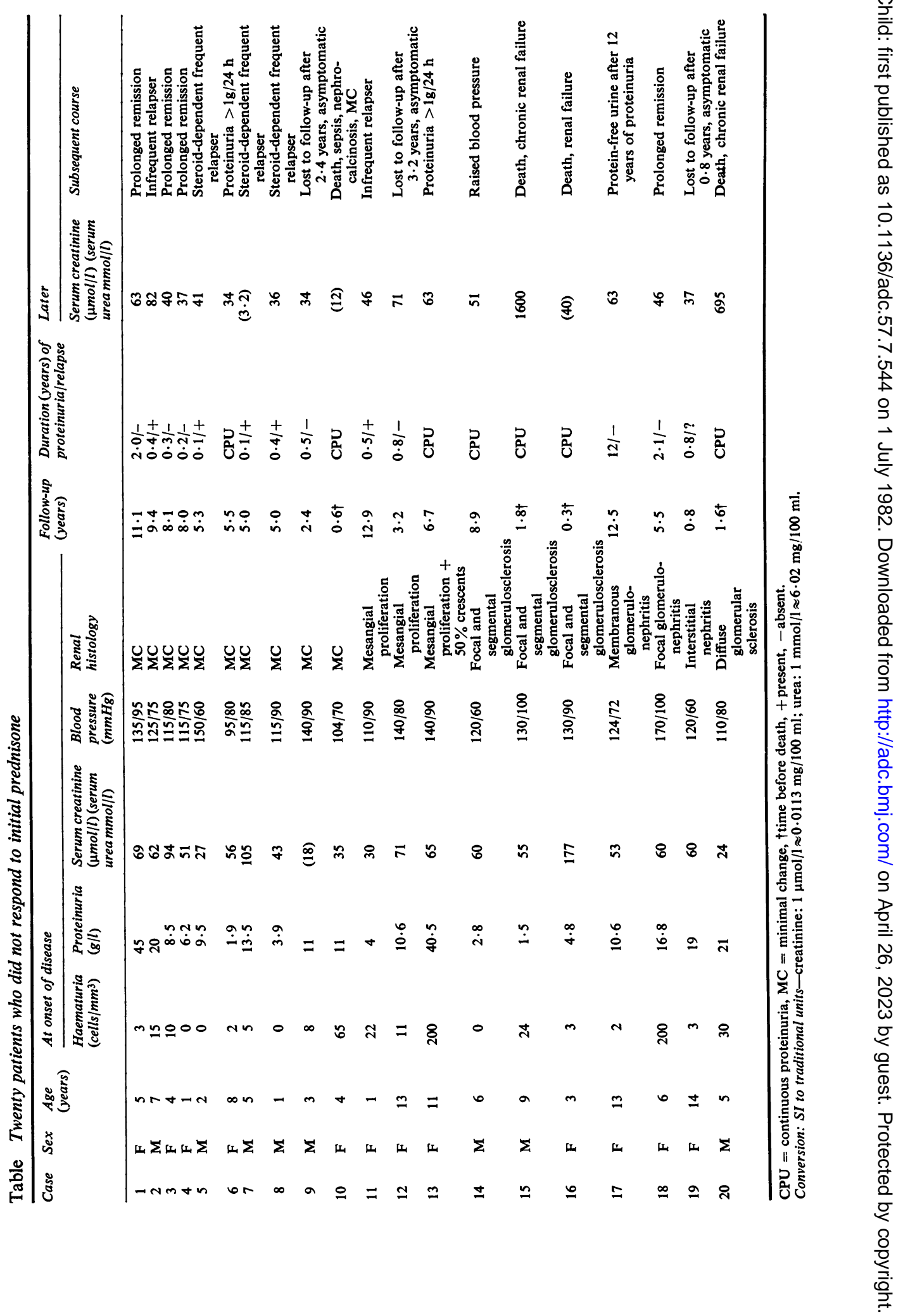


glomerulonephritis, and one focal and segmental proliferative glomerulonephritis.

\section{Subsequent clinical course}

At the time of this analysis (March 1981) after follow-up for at least 5 to a maximum of 14 years this group of 94 patients can be categorised as follows: 23 have never had a relapse, 21 have each had at least one relapse but never frequent ones, and 50 children have had a frequently relapsing phase at some stage of the disease-that is at least 2 relapses during any 6-month period. Eighteen of these 94 patients still have relapses (one or more relapses within 3 years) and one of them has developed persistent proteinuria. The last patient, a 13-year-old girl, had her first episode of NS at age 2 years, with focal and segmental proliferative glomerulonephritis in renal biopsy. She had a relapsing course of the disease until age 11, and then became hon-responsive both to prednisone and cyclophosphamide. She has a normal serum creatinine concentration and blood pressure. Two of 3 patients in whom the biopsy finding was mesangial proliferative glomerulonephritis have had frequent relapses but after a course of cyclophosphamide both have been well, one for 6 and the other for 10 years; the third has never relapsed in 12 years.

One patient has died; he had NS with a frequently relapsing course. He was killed by his mentally disturbed father while in remission. The remaining 92 patients have normal serum creatinine values and normal urinary findings, and their growth and blood pressure are normal.

Patients who did not initially respond to prednisone. Twenty of the 114 patients did not respond to the initial 8-week treatment with prednisone-that is they still had proteinuria after 8 weeks of treatment (Table). Ten children had minimal change histology but in the remaining children histology varied. Three of the children with minimal change histology were steroid-dependent frequent relapsers (Cases 5, 7, and 8); two of them (Cases 5 and 7) had a few days of protein-free urine at the onset of the disease but had proteinuria again 8 weeks after treatment started. A 4-year-old girl, with minimal change histology and continuous proteinuria (Case 10), died of sepsis and fluid and electrolyte disturbance 7 months after the onset of the disease.

Three children had mesangial proliferative glomerulonephritis and 3 had focal and segmental glomerular sclerosis; in the remaining children histology in the renal biopsy varied. Two children with focal and segmental glomerular sclerosis had progressively declining renal function, and both died; the third child has a normal serum creatinine value but a raised blood pressure and proteinuria of more than $1 \mathrm{~g}$ /day after 8.9 years of follow-up. A 5 -year-old boy also had progressive renal failure and died while on dialysis, his renal biopsy sample showed diffuse glomerular sclerosis at the onset of the disease.

Haematuria ( $>5$ cells $/ \mathrm{mm}^{3}$ ) at the onset of the disease was present in 10 of 20 children; three eventually died and one still has proteinuria. Thirteen children went into remission at a later stage (between 0.2 and 12.0 years after the initial findings of NS), 5 spontaneously, 4 during a repeated course of prednisone, 2 in response to cyclophosphamide, and 2 during treatment with cyclophosphamide and prednisone combined. Six have had no further relapses for at least 5 years.

Urine analysis and renal function estimated from serum creatinine values showed that the 13 patients who eventually went into remission have normal urine and serum creatinine values. In 7 other patients the proteinuria was not arrested; 4 of them have died, the remaining 3 have normal serum creatinine values although one has a high blood pressure.

\section{Discussion}

During the period 1967-76 any child with NS from a particular area of Finland was examined and treated at the Children's Hospital. Neither severity of the disease nor response to treatment affected selection, and this may explain why so many patients responded to prednisone and why so many had minimal changes in the biopsy compared with other series. ${ }^{15}$ Other but less likely explanations include differences in climatic or other geographical factors, type of infections, or genetic susceptibility, any one of which might modify the incidence of renal disease with an immunological pathogenesis.

All patients in this series have been regularly followed up; each has been seen in the outpatient department at least once a year even if he has been free of symptoms for several years. Only three of the 114 children did not regularly visit the outpatient department after becoming free of proteinuria.

Despite the fact that several series of childhood NS have been described ${ }^{2} 67$ the prognosis beyond childhood of an unselected group of patients may be unclear to some general pediatricians who take care of children with this disease. Our aim has been to give an outline of the prognosis for children with NS. The outcome can be related to the response to the initial prednisone treatment, and it is at that stage that the parents and child will need information about the nature of the disease. Children who are likely to need a renal biopsy and special management 
can be identified by their lack of response to the initial treatment with prednisone. Children with NS who respond to the first prednisone treatment by losing their proteinuria have a good prognosis (Figure). Of 94 such patients, one died accidentally, and only one did not respond to any attempt at treatment later in the disease. The remaining 92 children have normal urine analyses and normal renal function, and lead normal lives: 18 of them still have relapses. None of this group has a progressive renal disease-for example NS with focal and segmental glomerular sclerosis as the kidney biopsy finding, which may sometimes mimic minimal change nephropathy (International Study of Kidney Disease in Children, 1981, unpublished data). Thus the main risk seems to be from the side effects of the drugs, either of steroids or of cytotoxic drug therapy.

The steroid-responsive type of NS seems to be self-limiting and, if the onset is during childhood, the disease seems to be over or at least in prolonged remission before full adulthood.

Of the 20 children with continuing proteinuria after the initial prednisone treatment, 10 had minimal changes and the rest had varying histopathological changes in the renal biopsy samples. Although the outcome of children with minimal change nephropathy was generally favourable, one child died from sepsis. Renal biopsy should be done in children who initially do not respond to steroids, so as to distinguish patients with minimal change histology from those with other glomerular changes that are empirically associated with varying clinical courses and prognoses.

Children who do not respond to prednisone therapy and have other than minimal change nephropathy, 10 out of 114 in this series, have a bad prognosis; 3 have died and 1 has a high blood pressure and gross proteinuria. In these difficult cases, including responders who have frequent relapses, the examinations and follow-up should be performed at specialised nephrological centres, $\rho$ where all the necessary investigations can be performed and the type of treatment, or withholding of unnecessary therapy, can be planned.

The Sigrid Jusélius Foundation and Kidney Foundation provided generous financial support.

\section{References}

1 International Study of Kidney Disease in Children. $\vec{\circ}$ Report. Nephrotic syndrome in children: prediction of histopathology from clinical and laboratory characteristics at time of diagnosis. Kidney Int 1978; 13: 159-65.

2 Barnett H L. The natural and treatment history of glomerular diseases in children-what can we learn from international cooperative studies? In: Giovannetti $S$, o Bonomini V, D'Amico G, eds. Proceedings of the Sixth . International Congress of Nephrology, Florence 1975. Basel: Karger, 1976: 470-85.

3 International Study of Kidney Disease in Children. Report. The primary nephrotic syndrome in children. 음 Identification of patients with minimal change nephrotic syndrome from initial response to prednisone. $J$ Pediatr $1981 ; 98: 561-4$.

4 Churg J, Habib R, White R H R. Pathology of the nephrotic syndrome in children. A Report for the $\overrightarrow{0}$ International Study of Kidney Disease in Children. $\infty$ Lancet 1970; i: 1299-302.

5 Habib R, Kleinknecht C. The primary nephrotic syndrome of childhood: classification and clinicopathologic study of 406 cases. In: Sommers S C, ed. Pathology annual. Vol. 6. New York: Appleton-Century-Crofts, $1971: 417-74$

6 Siegel J S, Goldberg B, Krassner L S, Hayslett J P. Long $\mathbb{D}$ term follow-up of children with steroid-responsive nephrotic syndrome. J Pediatr 1972; 81 : 251-8.

7 Schwartz M W, Schwartz G J, Cornfeld D. A 16-year follow-up study of 163 children with nephrotic syndrome. Pediatrics 1974; 54: 547-52.

Correspondence to Dr Ollie Koskimies, Children's 융 Hospital, Department of Paediatrics, University of Helsinki, Stenbäckinkatu 11, SF-00290 Helsinki 29, Finland.

Received 28 September 1981 